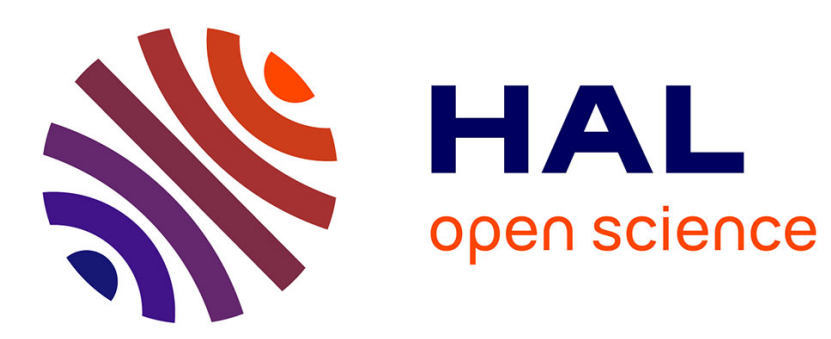

\title{
La nouvelle architecture constitutionnelle et les trois désamorçages de la vie politique marocaine
}

\author{
Jean-Noël Ferrié, Baudouin Dupret
}

\section{To cite this version:}

Jean-Noël Ferrié, Baudouin Dupret. La nouvelle architecture constitutionnelle et les trois désamorçages de la vie politique marocaine. Confluences Méditerranée , 2011, 78, pp.25-34. halshs00764212

\section{HAL Id: halshs-00764212 \\ https://shs.hal.science/halshs-00764212}

Submitted on 12 Dec 2012

HAL is a multi-disciplinary open access archive for the deposit and dissemination of scientific research documents, whether they are published or not. The documents may come from teaching and research institutions in France or abroad, or from public or private research centers.
L'archive ouverte pluridisciplinaire HAL, est destinée au dépôt et à la diffusion de documents scientifiques de niveau recherche, publiés ou non, émanant des établissements d'enseignement et de recherche français ou étrangers, des laboratoires publics ou privés. 
Ferrié \& Dupret, «La nouvelle architecture constitutionnelle et les trois désamorçages »,

Confluences Méditerranée

\section{La nouvelle architecture constitutionnelle et les trois désamorçages de la vie politique marocaine}

On serait spontanément tenté de lire la réforme constitutionnelle en cours comme la conséquence des pressions de la rue et, partant, comme une «constitution octroyée » dissimulant plus ou moins bien que le régime aurait tout bonnement «lâché du lest ». Si cette lecture respecte la succession des événements tels, du moins, qu'ils furent médiatisés - la manifestation du 20 février, le discours du roi du 9 mars annonçant la préparation d'une nouvelle Constitution et l'adoption de celle-ci le $1^{\text {er }}$ juillet -, elle ignore la séquence plus longue dans laquelle s'inscrit la réforme constitutionnelle et, par conséquent, surévalue largement le rôle joué par pression de la rue et du Mouvement du 20 février.

Disons-le tout net, la temporalité des « révolutions arabes » ne s'applique pas au Maroc et le «Mouvement du 20 février » n'y a pas été une réplique du mouvement de la place Tahrir, même s'il a tenté de se glisser dans l'espace d'opportunité ouvert par celui-ci, profitant au passage de l'extrême complaisance des médias étrangers et, parfois, marocains le présentant comme un mouvement de fond de la jeunesse. Au moment de la première manifestation, organisée le 20 février, le Maroc est, en effet, depuis longtemps dans une dynamique de réforme. Il ne s'agit de pas de soutenir que cette dynamique est une dynamique de «transition démocratique ». On attend généralement par ce terme un processus pacifié, plus ou moins rapide, par lequel des gouvernants et leurs institutions cèdent la place à d'autres institutions et à d'autres gouvernants ; une partie des anciens gouvernants, généralement des «réformateurs", pouvant également se mêler aux nouveaux ou être à l'initiative du changement en se transformant d'abord eux-mêmes. Ce fut le cas de la transition espagnole, conduite par des réformateurs issus du franquisme ${ }^{1}$. Au Maroc, il est difficile de parler de transition démocratique, non pas parce que le régime ne se serait jamais libéralisé, mais parce qu'il n'a jamais été question de changement de régime au sens de remplacer les institutions par d'autres. Selon la formule d'Abdallah Saaf, il est plus approprié de parler de transition politique, en ce sens que le régime demeure mais se modifie considérablement en s'incorporant de nouvelles élites ${ }^{2}$. En somme, il étend son assise au fur et à mesure qu'il se libéralise. Il est ainsi difficile d'établir une comparaison avec l'Egypte (comme on a plus ou moins tenté de le faire) où Hosni Moubarak a démissionné neuf jours avant la manifestation du 20 février. Il gouvernait depuis une trentaine d'année dans une ankylose grandissante. Ni en Egypte ni, du reste, en Tunisie, l'opposition et, plus largement, les partis politiques, à l'exception du parti présidentiel, ne participaient à la vie politique. Au mieux la décoraient-ils. Bref, la situation y était bloquée et, partant, potentiellement délétère. Ce n’était nullement le cas au Maroc.

Par conviction et par opportunité (les deux motifs sont assez difficiles à partager, puisque l'intérêt que l'on a à faire quelque chose n'exclut pas qu'on soit convaincu de son bien-fondé), la monarchie n'a jamais entièrement bloqué ni le jeu institutionnel ni le jeu partisan. Après la Constitution autoritaire de 1970, il y eut la Constitution de 1972 puis l'importante révision de 1996. 1972 marquait la fin de l'illusion dramatique qu'il était possible de gouverner dans le mépris des partis. Deux tentatives successives de coup d'Etat avaient montré au roi Hassan II que l'appareil sécuritaire, sur lequel il espérait établir durablement son pouvoir, pouvait lui faire défaut. Il décida alors de réintroduire les partis dans le jeu politique. Il s'agissait de ne pas avoir

\footnotetext{
${ }^{1}$ Colomer, J., Games Theory and the Transition to Democracy : The Spanish Model, Londres, Edward Elgar Publishing, 1995 ?

2 Tozy, M., Monarchie et politique au Maroc, Paris, Presses de Sciences-Po, 1999, p. 282.
} 
tous les œufs dans le même panier. 1996 préparait l'alternance consensuelle ${ }^{3}$ de 1998 et posait les fondements de la situation actuelle ${ }^{4}$. C'est en priorité par rapport à cette séquence longue qu'il faut considérer les changements actuels, si l'on souhaite éviter une mésinterprétation de la dynamique sociopolitique du Maroc et de la logique de l'actuelle architecture constitutionnelle.

Nous soutenons donc que la réforme constitutionnelle est la poursuite et l'amplification d'une dynamique inaugurée par deux désamorçages, l'un principalement politique, à partir des années de 1990, et l'autre principalement social à partir de 1999. Nous décrirons, tout d'abord, les deux premiers désamorçages. Nous montrerons, ensuite, comment le discours du roi du 9 mars 2011 a opéré un double désamorçage : il a désamorcé le «Mouvement du 20 février » tout en l'utilisant afin de désamorcer les conservatismes opposés à la transition politique. Nous décrirons, enfin, la logique de la nouvelle architecture constitutionnelle et le pari qu'elle fait de la mise en place d'un « Cercle vertueux ».

\section{Les deux premiers désamorçages}

Le premier désamorçage a consisté dans l'intégration gouvernementale des partis de l'opposition appartenant à la Koutla . Cette intégration présentait d'évidentes limites, mais elle n'en a pas moins modifié profondément la scène politique en créant un consensus manifeste sur la monarchie et en débloquant (les deux choses étant partiellement liées) l'accès aux fonctions publiques pour toutes une partie de l'élite oppositionnelle. Le roi acceptait que la Primature aille à l'un de ses plus anciens opposants, Abderrahman El Youssoufi, Secrétaire général de l'USFP (Union socialiste des forces de progrès). En retour, celui-ci acceptait une limitation de ses droits à désigner l'ensemble des ministres de son gouvernement. Il s'agissait de l'Intérieur, de la Justice, des Affaires étrangères et des Affaires religieuses. Le roi circonscrivait ainsi l'autonomie de son Premier ministre à la politique économique et sociale. Ce partage n'avait pas seulement pour but de préserver la prééminence royale mais aussi d'amorcer le tournant social d'un régime qui ne s'était jusqu'alors guère préoccupé de développement humain. Cette «alternance » a notamment été rendue possible par une réorientation notable de la politique du précédent souverain en ce qui concerne les droits de l'homme, qu'il avait longtemps maltraité. C'est ainsi qu'il crée le Conseil consultatif des droits de l'homme, en 1990, puis un ministère des Droit de l'homme, en 1993. Dans le domaine social, il installe, en 1990, un Conseil national de la jeunesse et de l'avenir, dont le secrétariat général fut confié à Habib El Malki, l'un des membres dirigeant de l'USFP alors dans l'opposition.

Le deuxième désamorçage a consisté dans l'attention portée par le nouveau souverain, dès son accession au trône, au développement humain. On sait qu'il en a fait son «chantier de règne ». Il crée, en 1999, l'année de son accession au trône, la Fondation Mohammed V pour la Solidarité et, en 2005, il lance l'Initiative nationale pour le développement humain (INDH). A ceci s'ajoute, la lutte contre les disparités régionales ainsi que l'effort dans le développement des infrastructures et dans le désenclavement du monde rural. On peut discuter de l'importance des résultats mais non les nier. Ils sont évidents, notamment dans les espaces ruraux. De même que l'est l'intérêt du souverain pour ces questions. Indépendamment des résultats, cet intérêt manifeste et manifesté a un impact sur la manière dont la monarchie est perçue et, donc, sur sa popularité et sur sa légitimité. Le désamorçage politique et social a aussi eu comme conséquence d'élargir l'espace de discussion seulement entrouvert à la fin du règne d'Hassan II.

\footnotetext{
${ }^{3}$ Ferrié, J.-N., « Maroc : la mise en place de l'alternance », Annuaire de l'Afrique du Nord 1998. Paris, CNRS-Editions, 2000.

${ }^{4}$ Rerhaye, N. et H. El Malki, Une parenthèse désenchantée. Une alternance marocaine, Casablanca, La croisée des chemins, 2011.

${ }^{5} \mathrm{Ou}$ « Bloc démocratique », coalition de partis de gauche (USFP, PPS, OADP) et d'un parti conservateur (Istiqlal).
} 
Cette ouverture a, certes, pris un aspect paradoxal, puisque l'implication dans l'activisme « civil», voire dans l'activité politique elle-même - participer aux élections et faire campagne - s'est accompagné d'une large dépolitisation, c'est-à-dire d'un désinvestissement vis-à-vis de l'engagement partisan et d'un désenchantement vis-à-vis des solutions politiques globales ${ }^{6}$; toutefois, l'espace public n'en a pas moins été occupé. On n'a pas attendu le 20 février pour débattre et parler librement au Maroc. Certes, il y avait (et il y a) des lignes rouges, mais il suffit de comparer les lignes rouges flottantes et circonscrites du règne actuel à celles, rigides, du règne précédent pour avoir une idée de l'ampleur du chemin parcouru. En douze ans, le Maroc a beaucoup changé.

En accompagnement de ce désamorçage social, Mohammed VI s'est attaché à promouvoir le pluralisme de la société marocaine ${ }^{7}$. Ce fut, tout d'abord, la reconnaissance de l'identité berbère avec la création de l'Institut royal de la culture amazighe (IRCAM), en 2001, puis le soutien apporté aux festivals de musique, notamment de "musique du monde », qui se caractérisent, en outre, par une large tolérance et une faible intervention de la police en matière de consommation de drogue et de relations entre les $\operatorname{sexes}^{8}$. Pareillement, l'insistance sur une forme marocaine de l'islam, dans le discours du trône du 30 juillet 2003, met en valeur une alternative à la conception moniste que l'islamisme se fait de l'identité musulmane. On remarquera que le ministre des Affaires religieuses, Ahmed Toufiq est un historien, romancier et membre de la confrérie soufie Boutchichya. La réforme du code du statut personnel, en 2004, s'inscrit dans la même logique. Elle fait suite à un premier projet, promu par Saïd Saâdi, secrétaire d'Etat dans le gouvernement d'alternance en 1998, et abandonné à cause de la vaste campagne menée contre lui par les conservateurs comme du peu d'empressement de sa propre majorité à le soutenir.

\section{Le double désamorçage de 2011}

C'est sur la base de ces changements consistants - même s'ils connaissent évidemment des limites - et non à cause des manifestations du 20 février qu'est intervenue la réforme de la Constitution. Les mutations qu'a connues la société marocaine n'avaient jusqu'à présent pas trouvé d'expression institutionnelle qui puisse donner une idée de l'orientation que le régime allait prendre par rapport à elles : allaient-elles s'inscrire, oui ou non, dans un tournant résolument démocratique? De nombreuses évolutions institutionnelles, on vient de citer les principales, indiquaient, depuis longtemps, une réorientation de la gouvernance. Plus récemment, les travaux des la Commission consultative de la régionalisation signifiait et impliquait un changement en profondeur des relations entre gouvernants et gouvernés comme la création du Conseil national des droits de l'homme et du Conseil économique, social et environnemental. C'est, du reste, en partant de la régionalisation que le souverain, dans son discours du 9 mars, annonçait la réforme constitutionnelle. C'est cette annonce, qui par son ampleur réformatrice, opère alors un troisième désamorçage plus complexe. Elle désamorce l'incertitude sur l'orientation du régime et la volonté du roi d'aller jusqu'au bout de la logique dans laquelle il s'était inscrit. En d'autres termes, elle désamorce le Mouvement du 20 février, en proposant une réforme majeure de l'architecture constitutionnelle, c'est-à-dire de l'organisation de la relation gouvernants/gouvernés. De fait, les semaines suivantes montrèrent la difficulté de celui-ci à rebondir sur d'autres demandes consensuelles; sa capacité à mobiliser se résorbe, comme en témoigne sa relative radicalisation,

\footnotetext{
6 Voir Bennani-Chraibi, M., «Représenter et mobiliser dans l'élection législative au Maroc », dans M. BennaniChraïbi, M. Catusse et, J.- C. Santucci, dir., Scènes et coulisses de l'élection au Maroc. Les Législatives 2002, Paris, IremamKarthala, 2004 ; "Actes de vote et d'abstention à Casablanca », dans M. Bennani-Chraibi, M. Catusse et, J.- C. Santucci, dir., Scènes..., op. cit.

${ }^{7}$ Labdaoui, A., « Cambiamento di regimo senza cambiamento di regime. Il caso del Marocco », dans A. Baldinetti et A. Maneggia, dir., Processi politici nel Mediterraneo : dinamiche e prospettiva, Perouse, Morlacchi, 2009.

${ }^{8}$ Madjouli, Z., Trajectoire des musiciens Gnawa. Approche ethnographique des cérémonies domestiques et des festivals de Musiques du monde, Paris, L'Harmattan, 2008.
} 
au fur et à mesure que l'on s'approche de la date du référendum constitutionnel, ainsi que le jeu compensatoire consistant à s'allier de plus en plus nettement avec les islamistes du mouvement Justice et Bienfaisance (al-adl wa al-ihsan), qui passent pour avoir des assisses populaires qui lui font défaut. En fait, le Mouvement du 20 février a surtout constitué une opportunité pour la monarchie : celle de pouvoir accélérer les réformes en se fondant sur une demande explicite et en bénéficiant de la médiatisation d'un mouvement qui, bien que ne représentant précisément pas "la population», a réussi à s'imposer comme exprimant certaines de ses attentes. Les réformes apparaissaient alors comme une nécessité politique consensuelle et, implicitement, comme le moyen d'éviter les crises majeures que connaissaient d'autres pays de la région. Techniquement, il s'agit d'une manœuvre héresthétique, au sens de William Riker, c'est-à-dire d'une manœuvre destinée à présenter un choix comme évident et nécessaire sans avoir à entrer dans une défense argumentée de celui-ci'. La manœuvre a permis d'accomplir le deuxième volet du désamorçage, puisqu'elle a désamorcé les conservatismes inhérents à la vie sociale en apportant une justification évidente de la réforme constitutionnelle.

La question de la justification est, en effet, particulièrement importante : de nos jours, on ne réforme plus - même pour le mieux - sans consentement et sans participation. Paradoxalement, ce point n'apparaît pas toujours aux partisans de la démocratie maximale et immédiate. Une partie de la classe politique peut craindre les réformes, même les réformes démocratiques, parce qu'elles impliquent des changements et donc une prise de risque. Une partie de la population - et sans doute la partie de loin la plus importante - peut être fermement attachée à la Commanderie des croyants, tout simplement parce que c'est à la fois un rappel de l'identité musulmane du pays et de la particularité de cette identité. Une autre partie de la population peut être réticente à ce que le berbère devienne langue officielle. Une autre, encore, peut voir d'un mauvais œil l'affirmation de l'égalité entre hommes et femmes. Malgré ces divergences (et l'on pourrait en citer d'autres), de nombreux activistes et beaucoup de commentateurs se comportent vis-à-vis de la réforme constitutionnelle comme si elle ne mettait en cause que deux parties: les gouvernants, qui voudraient conserver tous les pouvoirs ou presque, et les gouvernés, qui les réclameraient à bon droit; mais cette vision horizontale et extrêmement réductrice et simplifie excessivement les lignes de partages. Il y a des opinions et des intérêts différents dans la société comme dans la sphère politique et la Constitution tente, d'abord, d'organiser la cohabitation et le dialogue entre ces opinions et ces intérêts. Une constitution, en effet, n'est pas qu'une réglementation de la distribution du pouvoir ; c'est aussi, et peut-être surtout dans nos époques démocratiques, un moyen d'administration pacifique, sinon toujours consensuel, des équilibres collectifs. Bref, une constitution ne doit pas seulement être acceptable par les plus libéraux. Elle doit l'être par tous les autres qui doivent s'y retrouver et dont les réticences doivent être vaincues.

\section{La nouvelle architecture constitutionnelle}

La nouvelle Constitution présente trois caractéristiques essentielles : la délimitation d'un large périmètre d'action en faveur du chef du gouvernement qui disposera des moyens nécessaires pour mener à bien sa tâche et, surtout, pour contrôler la majorité parlementaire le soutenant ; l'affirmation des pouvoirs d'arbitrage et d'influence du souverain; la mise en place d'instances indépendantes responsables de la protection et du développement des droits. Cette séparation vise moins à séparer l'Exécutif, le Législatif et le Judiciaire qu'à délimiter les sphères d'influences de trois bloc fonctionnels.

C'est ainsi que le gouvernement et le Parlement sont fonctionnellement liés bien plutôt qu'ils ne s'opposent ou ne s'équilibrent. Le choix du chef du gouvernement se fait nécessairement

\footnotetext{
${ }^{9}$ Riker, W., The Art of Political Manipulation, Yale, Yale University Press, 1986.
} 
parmi les membres du parti arrivé en tête. C'est une logique parlementaire. Il dispose du droit de dissolution. C'est la rationalisation du parlementarisme, d'autant plus nécessaire que les gouvernements marocains ont toujours été de coalition. Le droit de dissolution contribuera à faire du chef du gouvernement le véritable " patron» de sa majorité. Celui-ci disposera en outre de la possibilité d'engager la responsabilité du gouvernement sur un projet de loi, ce qui est un moyen de contrainte fort vis-à-vis du Parlement, renverser le gouvernement signifiant presque à coup sûr se retrouver devant les électeurs. Le reste dépendra de la loi électorale. En elle-même, ces mesures établissent déjà les fondations d'un système parlementaire fort ; mais, ce système ne représente qu'une composante de l'architecture constitutionnelle, là où, habituellement, il en représente l'essentiel. De ce point de vue, la nouvelle Constitution est réellement innovante. Du reste, elle diffère largement des précédentes qui visaient, en effet, avant tout à protéger la monarchie par rapport au gouvernement et au Parlement (encore qu'elle ne risquât pas grandchose). Celle-ci vise, au contraire, à consolider un espace de gouvernement autonome dans lequel le Chef du gouvernement est, de facto, directement responsable devant les gouvernés, par l'intermédiaire des élections. C'est sa désignation obligatoire parmi les représentants du parti arrivé en tête aux élections qui établit cette logique, somme toute proche des parlementarismes britannique et allemand où voter pour un parti, c'est en même temps choisir le chef de l'Exécutif. Les électeurs sauront, en effet, qui ils porteront à la tête du gouvernement et celui-ci saura qu'il ne pourra y demeurer qu'avec leur soutien, puisque le roi ne peut le nommer librement (ou le renvoyer) et qu'il ne pourra pas espérer conserver son poste même avec le soutien d'une coalition, si son parti n'arrive pas en tête. Il s'agit d'une arme très forte donnée aux électeurs en contrepartie des pouvoirs concédés au Chef du gouvernement.

Cette arme apparaît destinée (ou pourrait être destinée, mais il existe de bonnes raisons de penser que c'est volontaire) à synchroniser politics et policies $^{10}$, c'est-à-dire l'activité politique ellemême (politicienne, partisane...) et la conduite des politiques publiques ; en d'autres termes, il s'agit de sortir de la situation où des politiques publiques sont promues tout en n'étant pas ou suffisamment effectives. C'est un problème constant dans la conduite des politiques publiques que celui des multiples courroies de transmission de l'appareil étatique et des déperditions qui en découlent. Dans la conduite des politiques publiques, le Maroc a jusqu'alors privilégié l'action consensuelle (mise en place d'instances consultatives d'évaluation, négociation avec les groupes intéressés...) ou «directe ». L'action directe est illustrée par l'intervention du roi dans la mise en place d'infrastructures ou par le renvoi des gouverneurs accomplissant mal leurs tâches. Toutefois, ce dernier mode d'action n'est pas efficace que dans certains domaines. Le système sanitaire, la protection sociales, la justice et l'enseignement n'en bénéficient pas. Pourquoi ? Parce que la mise en place d'infrastructures ne réclame pas de réviser un système mais seulement d'exercer une certaine pression sur celui-ci. En revanche, obtenir (par exemple) que la corruption cesse, c'est s'attaquer à un équilibre complexe et collaboratif. C'est prendre en compte à la fois la rémunération des agents publics, le motif de leur dévouement au régime, les raisons de la crainte qu'ils inspirent, l'attitude des citoyens comme la conception qu'ils se font du rapport à l'administration et, plus largement, au civisme. C'est aussi prendre en compte l'attitude déviante d'une multitude d'acteurs. Dans le domaine sanitaire, vouloir mettre fin au mauvais fonctionnement des hôpitaux, c'est aussi prendre en compte le salaire des fonctionnaires et la carrière des médecins, qui considèrent leur affectation dans un hôpital éloigné des grandes villes comme un mauvais moment à passer, notamment parce qu'ils ne peuvent pas doubler le salaire de leur emploi public par un salaire obtenu en travaillant dans une clinique privée. Dans le domaine de la protection sociale, dont le chantier a été ouvert dès 1998, il ne s'agit pas seulement de mettre en place un bon système, il s'agit de trouver une péréquation entre ce que les entreprises peuvent donner, l'effort que l'Etat peut consentir et la participation des bénéficiaires,

\footnotetext{
${ }^{10}$ Sur cette distinction, voir Camau, M. et G. Massardier, «Revisiter les régimes politiques », dans Camau, M. et G.
} Massardier, dir., Démocraties et autoritarismes. Fragmentation et bybridations des régimes, Paris, Karthala, 2009. 
chacun de ces trois partenaires étant pris dans des systèmes de contraintes propre. En d'autres termes, il est question de parvenir à mobiliser durablement différentes catégories d'acteurs de l'action publique. Ce n'est jamais facile, notamment parce que la stabilité des gouvernants est souvent tributaire d'immobilismes impliquant de ne pas malmener trop de monde, notamment parmi les agents de l'Etat, qui assurent, chacun à leur place, la stabilité du régime à défaut de nécessairement assurer le règne de l'Etat de droit. Dans un régime " compact», il est difficile au Chef de l'Etat de prendre ce risque, puisqu'il bénéficie paradoxalement de l'immobilisme contre lequel il lutte. En revanche, en créant un Chef du gouvernement et un Exécutif distinct du roi, la réforme constitutionnelle a créé un acteur qui a intérêt à malmener les agents publics pour conserver son poste et la prééminence de son parti. La monarchie n'endosse pas, elle, le coût de ces (éventuelles) transformations mais les arbitre.

La logique parlementaire du texte est, en effet, bornée par les attributions du souverain, mais elles ne la remettent pas en cause. Il faut les concevoir comme étaient conçus les pouvoirs présidentiels au début de la Vème République française (entre 1958 et 1962), à l'époque où le président n'était pas aussi le chef effectif de la majorité parlementaire, mais où il disposait en même temps d'une forte légitimité historique. Ses pouvoirs étaient considérés comme le moyen de mieux protéger les intérêts fondamentaux du pays en ne les plaçant pas au centre des négociations partisanes. Cette conception n'est pas dénuée de pertinence. Elle est proche de l'esprit du constitutionnalisme qui consiste à ne pas laisser toutes les décisions dépendre du fait majoritaire. Dans le même ordre d'esprit, l'instauration d'une cour constitutionnelle, adossée à la large déclaration des droits figurant dans la Constitution et à laquelle le citoyen pourra s'adresser, garantit l'existence d'une sphère indépendante de protection et de développement des droits, échappant elle aussi aux aléas de la gouvernance et des conservatismes électoraux. Ce qu'il reste à faire, maintenant, c'est d'habiter et de faire vivre cette belle architecture.

Or, c'est ici que les choses se compliqueront probablement. Les incontestables avancées que contient le texte dépendront largement de leurs mises en applications et notamment des lois organiques et des jugements de la Cour constitutionnelle. Beaucoup des innovations libérales de celui-ci sont, en effet, seulement posées en principe. Par exemple, la Constitution dispose que « la Cour constitutionnelle est compétente pou connaitre d'une exception d'inconstitutionnalité soulevée au cours d'un procès» (art. 113). C'est une loi organique qui fixera les modalités d'application de cet article. Or, la loi peut être restrictive en imposant, comme en France, un véritable filtre ou libérale, en s'en abstenant. Ce sont donc les députés, après l'élection législative de l'automne, qui décideront si les disposition libérales de la Constitution seront développés ou, au contraire, restreintes. Dans le même ordre d'idées, il est dit que «Le droit à la vie est le droit premier de tout être humain » (art. 20). Cette disposition peut aussi bien servir à bloquer le droit à l'avortement qu'à permettre l'abolition de la peine de mort (qui n'est plus appliquée mais demeure). On pourrait multiplier les exemples. Dans ce domaine, la Cour constitutionnelle aura un rôle déterminant, mais cela dépendra de sa composition. On peut avoir deux interprétations de cette indétermination, du reste relative, du texte constitutionnel : celle consistant à en déduire qu'il ne va pas assez loin et celle consistant à considérer qu'il créé de vrais potentialités laissant aux acteurs le soin de s'entendre sur le contenu exact de celles-ci. Peut-être est-ce la seule façon raisonnable de faire pour que la diversité des opinions et des intérêts soit respectée. Mais c'est en définitive à quoi servent les architectures constitutionnelles : à créer des comportements collectifs à même de les consolider. Cela s'appelle le cercle vertueux.

Jean-Noël Ferrié et Baudouin Dupret Directeurs de recherche au CNRS, Centre Jacques Berque, Rabat. 